\title{
CTP and DCI: We Need Clarification
}

\author{
Thomas Mattingly ${ }^{1}$ (i)
}

Published online: 18 July 2017

(C) Springer Science+Business Media, LLC 2017

The work "Changes in Cerebral Perfusion with Induced Hypertension in Aneurysmal Subarachnoid Hemorrhage: a Pilot and Feasibility Study" by Murphy et al. [1] is a retrospective investigation regarding the utility of computed tomography perfusion (CTP) before and after treatment of delayed clinical ischemia (DCI) via induced hypertension (IH). This work builds on an extensive history of treatment of DCI using "triple $\mathrm{H}$ therapy" by applying an efficient and widely available method of measuring cerebral blood flow. The literature surveyed in this paper reflects the current conflict regarding treatment of DCI: namely, DCI is linked to worse outcomes after subarachnoid hemorrhage (SAH), but treatment of DCI has not been shown to improve outcomes.

This study is a pragmatic retrospective look at a realworld SAH service. Patients received a CTP soon after arrival, then again during the period when DCI is most likely to appear. Of the three measurements obtained in CTP, mean transit time (MTT) is the most useful. In fact there is a predictive effect of prolonged MTT on early CTP for DCI later. In the DCI group, MTT drops significantly after institution of IH. However, there was no difference in infarct or poor outcome over the group that did not develop DCI.

The "triple H" therapy for the treatment of DCI includes, hypervolemia, hypertension and hemodilution. Increasing recognition of complications from "triple $\mathrm{H}$ " therapy has led to a renewed evaluation of the role of each

Thomas Mattingly

tmattingly206@gmail.com

1 Neurosurgical Associates, Johnston Willis Hospital, Richmond, VA, USA

of the three components. As a result, 2 of the 3 "H"s have lost importance, leaving induced hypertension as the mainstay of management of DCI [2]. "Triple H" therapy has been evaluated both as a treatment and as prophylaxis for DCI, but without consensus [3]. This paper gets to that central question. By design, IH in this paper is "treatment" for clinical DCI. However, the finding of early MTT prolongation well before the development of symptoms raises the possibility that IH may have a role in "prevention" of DCI as well as treatment. Perhaps there is a role for hypertension immediately after the aneurysm is secured, and this might be evaluable using CTP.

Although DCI is the leading cause of death and disability in treated aneurysmal SAH, the use of "triple $\mathrm{H}$ " has not necessarily yielded better outcomes. It is unclear what exactly is changing in the physiology of aneurysmal SAH when DCI is "treated." Intuitively, this appears to be a change in cerebral blood flow (CBF). Numerous methods including PET, SPECT, MRI, and now CTP have attempted to identify this signature [4]. CTP, with its practical advantages of widespread availability and rapid acquisition, has received more attention recently with mixed results $[5,6]$. Murphy et al. indicate that $\mathrm{CBF}$ may be the wrong signature. It appears that MTT, not CBF, is the key component both to identify those more likely to develop DCI, but also for monitoring the effect of treatment. The authors were able to show a significant change with MTT with one-tenth the patients that have been thought to be needed for a trial of $\mathrm{IH}$ if one is looking for changes in $\mathrm{CBF}$ [7].

This paper, like others, did not find clinical differences in the DCI versus the non-DCI cohorts. This may simply be that IH improved outcomes in the DCI cohort to that of the non-DCI cohort. The authors state that randomization of patients with DCI to IH treatment is not ethical at their institution. They also cite mounting evidence of both harm 
and lack of clinical efficacy for current DCI therapy. Therefore, clinicians are ethically obligated to objectively evaluate the treatment (or prevention) of DCI just as any other therapy. A randomized trial (HIMALAIA) to evaluate IH in DCI recently closed due to lack of recruitment but this should not be the final word on the topic [8]. I hope this paper, with its findings of MTT as a sensitive measure for both selecting patients and evaluating therapeutic response, serves as inspiration to pursue this question further.

\section{References}

1. Murphy A, de Oliveira Manoel AL, Macdonald RL, et al. Changes in cerebral perfusion with induced hypertension in aneurysmal subarachnoid hemorrhage: a pilot and feasibility study. Neurocrit Care. 2017; . doi:10.1007/s12028-017-0379-6.

2. Treggiari MM, Deem S. Which $\mathrm{H}$ is the most important in triple-H therapy for cerebral vasospasm? Curr Opin Crit Care. 2009;15(2):83-6.

3. Dankbaar JW, Slooter AJ, Rinkel GJ, et al. Effect of different components of triple- $\mathrm{H}$ therapy on cerebral perfusion in patients with aneurysmal subarachnoid haemorrhage: a systematic review. Crit Care. 2010;14:R23.

4. Keyrouz SG, Diringer MN. Clinical review: prevention and therapy of vasospasm in subarachnoid hemorrhage. Crit Care. 2007;11:220.

5. Mir DI, Gupta A, Dunning A, et al. CT perfusion for detection of delayed cerebral ischemia in aneurysmal subarachnoid hemorrhage: a systematic review and meta-analysis. AJNR Am J Neuroradiol. 2014;35:866-71.

6. Cremers $\mathrm{CH}$, van der Schaaf IC, Wensink E, et al. CT perfusion and delayed cerebral ischemia in aneurysmal subarachnoid hemorrhage: a systematic review and meta-analysis. J Cereb Blood Flow Metab. 2014;34:200-7.

7. Gathier CS, Dankbaar JW, van der Jagt M, et al. Effects of induced hypertension on cerebral perfusion in delayed cerebral ischemia after aneurysmal subarachnoid hemorrhage: a randomized clinical trial. Stroke. 2015;46:327-81.

8. Gathier CS, van den Bergh WM, Slooter AJ, et al. HIMALAIA (Hypertension Induction in the Management of AneurysmaL subArachnoid haemorrhage with secondary IschaemiA): a randomized single-blind controlled trial of induced hypertension versus no induced hypertension in the treatment of delayed cerebral ischemia. Int J Stroke. 2014;9:375-80. 